\title{
Compartment-Specific Gene Regulation of the CAR Inducer Efavirenz In Vivo
}

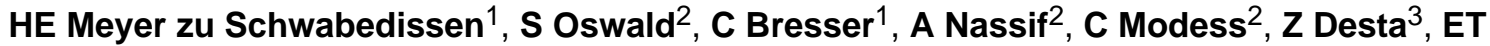 \\ Ogburn ${ }^{3}$, M Marinova $^{4}$, D Lütjohann ${ }^{4}$, C Spielhagen ${ }^{5}$, M Nauck ${ }^{5}$, HK Kroemer ${ }^{1}$, and W \\ Siegmund ${ }^{2}$ \\ ${ }^{1}$ Department of Pharmacology, University of Greifswald, Greifswald, Germany \\ ${ }^{2}$ Department of Clinical Pharmacology, University of Greifswald, Greifswald, Germany \\ ${ }^{3}$ Division of Clinical Pharmacology, Department of Medicine, Indiana University School of \\ Medicine, Indianapolis, Indiana, USA \\ ${ }^{4}$ Department of Clinical Chemistry and Clinical Pharmacology, University of Bonn, Bonn, \\ Germany \\ ${ }^{5}$ Department of Clinical Chemistry and Laboratory Medicine, University of Greifswald, Greifswald, \\ Germany
}

\begin{abstract}
Nuclear receptors such as the constitutive androstane receptor (CAR) are central factors that link drug exposure to the activities of drug metabolism and elimination. In order to determine the in vivo effects of efavirenz, a CAR activator, the expression of target genes was determined in duodenal biopsies obtained from 12 healthy volunteers before treatment and after 10 days of treatment with efavirenz; concomitant administration of the cholesterol inhibitor ezetimibe produced no significant difference. However, in in vitro studies, efavirenz significantly increased CYP2B6 expression in several cell types, suggesting that the drug transactivates CAR. This hypothesis is supported by our findings that there is significant induction of CAR target genes in in vivo peripheral blood mononuclear cells (PBMCs) isolated from healthy volunteers treated with multiple doses of efavirenz. The impact of efavirenz on hepatic metabolism in vivo was confirmed by significant changes in plasma $4 \beta$-hydroxycholesterol and bilirubin levels and the area under the curve (AUC) of efavirenz. induction of CYP2B6 mRNA expression correlated with the decrease in the AUC of efavirenz $(r=0.61 ; P=0.036)$. Taken together, our results provide evidence that efavirenz exerts compartment-specific inductive capacity in vivo.
\end{abstract}

\footnotetext{
(C) 2012 American Society for Clinical Pharmacology and Therapeutics

Correspondence: HE Meyer zu Schwabedissen (henriette.meyerzuschwabedissen@uni-greifswald.de).

SUPPLEMENTARY MATERIAL is linked to the online version of the paper at http://www.nature.com/cpt AUTHOR CONTRIBUTIONS

H.E.M.z.S. wrote the manuscript, designed research, performed research, and analyzed data. S.O. wrote the manuscript, performed research, and analyzed data. C.B. performed research and analyzed data. A.N. performed research. C.M. performed research. Z.D. wrote the manuscript, performed research, and analyzed data. E.T.O. performed research and analyzed data. M.M. performed research and analyzed data. D.L. wrote the manuscript, performed research, and analyzed data. C.S. performed research. M.N. performed research. H.K.K. wrote the manuscript and contributed new reagents/analytical tools. W.S. wrote the manuscript, designed research, and contributed new reagents/analytical tools.
}

CONFLICT OF INTEREST

M.N. is an adviser to Becton Dickinson. The other authors declared no conflict of interest. 
The processing of exogenous and endogenous compounds in the liver is based on cellular mechanisms such as transmembrane transport and enzymatic metabolism. Accordingly, expression and activity of genes involved in this network are prerequisites for drug disposition. Since the first description of the nuclear receptor PXR (pregnane X receptor) as a sensor that regulates expression of CYP3A4 in response of the cellular load of activating xenobiotics, ${ }^{1}$ our understanding of the role of nuclear receptors has evolved extensively. It is known that PXR regulates a network of genes involved in drug metabolism, including phase I and phase II enzymes such as cytochrome P450 enzymes, ${ }^{1-3}$ members of the UGT family, ${ }^{4,5}$ and sulfotransferases ${ }^{6,7}$ as well as drug transporters such as OATP1A2 (ref. 8) and ABCB $1 .{ }^{9}$ As a result, PXR influences bioavailability and systemic elimination of many compounds and plays a major role in mediating induction based drug-drug interactions. The discovery of PXR and its impact on drug disposition provided a mechanistic rationale for several clinically relevant drug-drug interactions. ${ }^{10}$ Among these are those reported in combination with St. John's wort. Hyperforin, a major constituent of this herbal extract, was identified as being a potent PXR inducer. ${ }^{11}$ Consequently, the treatment failures observed when highly metabolized drugs such as contraceptives, cyclosporine, or HIV protease inhibitors are administered in the presence of St. John's wort could be explained on a mechanistic basis. ${ }^{12}$

The constitutive androstane receptor (CAR) is another member of the nuclear receptor family, and its target genes broadly overlap those of PXR. CAR has been shown to regulate the expression levels of CYP3A4, CYP2B6, CYP2C9, ABCB1, and UGT1A1. ${ }^{13-16}$ Further evidence of the promiscuity of PXR and CAR is the finding that both receptors interact with the same response elements in target gene promoters. ${ }^{17}$ Although CAR had initially been reported to function as a constitutive active transcription factor, ${ }^{18}$ it is now widely accepted that there are several activating ligands. One of the most potent and specific agonists of CAR is the imidazole derivative 6-(4-chlorophenyl)imidazo[2,1- $\beta][1,3]$ thiazole-5carbaldehyde-O-(3,4-dichlorobenzyl)oxime (CITCO). ${ }^{19}$ In addition, there are a few drugs in clinical use that preferentially and directly interact with CAR and induce gene expression in vitro. These include statins, nevirapine, carbamazepine, phenytoin, and efavirenz. ${ }^{20,21}$ Changes in drug disposition as observed with efavirenz after chronic administration ${ }^{22}$ or in combination with statins ${ }^{23}$ have been explained on the basis of CAR-mediated induction of drug metabolism. However, little is known about the impact of these compounds on gene expression and activity in vivo.

The first purpose of this study was to test the induction capacity of efavirenz in vivo by measuring the expression of CAR target genes in the gastrointestinal tract as well as in peripheral blood mononuclear cells (PBMCs). The second objective was to monitor the influence of efavirenz on previously reported markers of hepatic metabolism. ${ }^{24}$ The results of our study provide strong evidence that CAR target genes are not induced in the intestine and that exposure to efavirenz significantly increases markers of hepatic drug metabolism and target gene expression in PBMCs. Importantly, induction in PBMCs was predictive of changes in efavirenz pharmacokinetics in vivo. The data presented here are part of a clinical study designed to identify not only the effects on target gene expression and activity but also the impact on the outcome after concomitant administration of ezetimibe. The study design of this trial is described in Supplementary Figure S1 online. The data on ezetimibe pharmacokinetics are summarized in a recent article by Oswald et al. $^{25}$

\section{RESULTS}

\section{Expression of CAR target genes in intestinal samples}

We examined mRNA in duodenal biopsy samples obtained from subjects before and after chronic treatment with efavirenz to investigate expression levels of CAR target genes. There 
was no significant induction of any of the genes presumed to be involved in the intestinal metabolism of ezetimibe, the drug assessed for drug-drug interaction in the clinical study (see Figure 1). No statistically significant induction was observed for $A B C B 1$ (normalized mRNA expression, mean $\pm \mathrm{SD}$ : pretreatment $1.76 \pm 2.04$; post-treatment $0.58 \pm 0.39$; Mann-Whitney test $P=0.099$ ), $A B C C 2$ (pretreatment $2.39 \pm 2.32$; post-treatment $4.19 \pm$ 6.16; Mann-Whitney test $P=0.694), U G T 1 A 1$ (pretreatment $1.66 \pm 1.51$; post-treatment $1.46 \pm 1.40$; Mann-Whitney test $P=0.645$ ), $C Y P 3 A 4$ (pretreatment $1.54 \pm 1.56$; posttreatment $0.61 \pm 0.37$; Mann-Whitney test $P=0.126$ ), and $C Y P 2 B 6$ (pretreatment $1.50 \pm$ 1.30; post-treatment $0.71 \pm 0.42$; Mann-Whitney test $P=0.126$ ). Similar results were obtained for the impact of long-term efavirenz treatment on protein expressions of UGT1A1 and ABCG2 (Figure 2a). We included $A B C G 2$ in our investigation because this gene has not been reported to be regulated by CAR. Although there seemed to be an induction of UGT1A1 protein in subject 7, this was not observed for other CAR target gene proteins such as those for ABCB1, ABCC2, and CYP3A4 in the same individual (Figure 2b). Similarly, in subject 12, we saw induction of ABCB1 protein, whereas no change was observed with regard to UGT1A1 and CYP3A4 protein expressions. It is noteworthy, however, that we were not able to determine protein expressions of all the genes ( $A B C B 1, A B C C 2$, and $C Y P 3 A 4)$ in all the samples because of limited availability of the protein extract from some individuals.

\section{Induction of CYP2B6 expression in Caco2 cells}

In order to test the ability of efavirenz to induce CAR target genes in intestinal cells, Caco2 cells transfected with CAR were treated with efavirenz and CITCO. As shown in Figure 3, there was a twofold increase in CYP2B6 expression at $48 \mathrm{~h}$ after transfection of CAR as compared with cells transfected with the vector control (mean CYP2B6 expression relative to vector control $\pm \mathrm{SD}: 2.30 \pm 0.26$; Student's $t$-test $P=0.001)$. Treatment with efavirenz $(10 \mu \mathrm{mol} / \mathrm{l})$ or CITCO $(1 \mu \mathrm{mol} / \mathrm{l})$ (significantly increased the expression levels of $C Y P 2 B 6$ in Caco 2 cells $(5.63 \pm 1.49$; Student's $t$-test $P=0.033$, and $8.29 \pm 3.33$; Student's $t$-test $P=$ 0.031 , respectively) (Figure 3a). Efavirenz treatment also significantly increased the expression of $C Y P 2 B 6$ in primary human hepatocytes (Figure $3 \mathrm{~b}$ ).

\section{Impact of efavirenz on CAR target genes in PBMCs in vitro}

To obtain insight into an additional pharmacologic compartment, we intended to assess the expression of CAR target genes in PBMCs isolated from healthy volunteers on the designated pharmacokinetic study days. In order to confirm that PBMCs are responsive to efavirenz treatment, we obtained PBMCs from two drug-naive individuals and treated the cells with efavirenz in vitro. On the basis of our findings of the maximum concentration of efavirenz determined in the steady state $(0.92 \pm 0.34 \mu \mathrm{g} / \mathrm{ml})$ (Table 1$)$ and the findings of Almond et al. that the cellular accumulation ratio ranges from 0.7 to 3.3 in PBMCs relative to the plasma levels of the compound, intracellular concentrations of efavirenz ranging from 2 to $10 \mu \mathrm{mol} / 1$ would be expected. ${ }^{26}$ Therefore, PBMCs were treated with $10 \mu \mathrm{mol} / \mathrm{l}$ of efavirenz and the expression levels of several previously reported CAR target genes were determined. Efavirenz treatment resulted in significant induction of $C Y P 2 B 6, A B C B 1$, and $U G T 1 A 1$ (Figure 4). Expression profiles of the same genes in primary hepatocytes are also provided for comparison (Figure 4).

\section{Expression of CAR target genes in PBMCs isolated from individuals treated with efavirenz and/or ezetimibe}

On the basis of our findings of responsiveness of mRNA expression of CYP2B6, ABCB1, and UGT1A1 to the CAR inducer efavirenz in vitro, we assessed PBMCs isolated from healthy individuals. We compared gene expressions in PBMCs isolated during period A (single-dose efavirenz) with those isolated during period B (multiple doses of ezetimibe) and 
period D (multiple doses of ezetimibe and efavirenz). As shown in Figure 5, we observed significant induction of CYP2B6 (Mann-Whitney test $P=0.002$ ) and $A B C B 1$ (MannWhitney test $P=0.001$ ) in the presence of multiple doses of efavirenz. However, no such impact was observed for the expression of UGT1A1 (Mann-Whitney test $P=0.100$ ). Importantly, we did not observe an impact on target gene expression after multiple doses of ezetimibe in treatment period B (Figure 5).

\section{Induction of hepatic metabolism in vivo}

Determination of the oxysterol $4 \beta$-hydroxycholesterol, a sensitive marker of CYP3A4 activity, revealed a nearly twofold induction after multiple doses of efavirenz ( $4 \beta$ hydroxycholesterol levels, mean $\pm \mathrm{SD}$ ng/ml, before efavirenz treatment $6.40 \pm 2.43$; and after efavirenz treatment $10.03 \pm 3.38$; Mann-Whitney test $P=0.006$ ), suggesting that the induction of CYP3A4 does indeed occur in vivo in the liver. Importantly, the levels of $4 \beta$ hydroxycholesterol and cholesterol were determined at several time points during the study, and significant induction of the $4 \beta$-hydroxycholesterol/cholesterol ratio was observed on study days 25 and 30 (Figure 6a). In addition, we observed significantly lower area under the curve (AUC) and elimination half-life $\left(t_{1 / 2}\right)$ after multiple-dose treatment with efavirenz than after single-dose treatment (Table 1). Long-term treatment with efavirenz significantly reduced total bilirubin levels (mean bilirubin levels \pm SD mg/dl before treatment $9.82 \pm$ 3.86; and after treatment 6.34 \pm 2.48 ; Mann-Whitney test $P=0.023$ ), suggesting induction of hepatic bilirubin metabolism, including enzymes and transporters involved in the elimination of bilirubin. No such impact was observed for treatment with multiple doses of ezetimibe, as determined from the pharmacokinetic parameters on study days 15 and 17 (Figure 6b).

Investigation of a possible correlation between the change in AUC of efavirenz and the change in the markers of hepatic drug metabolism, namely, $4 \beta$-hydroxycholesterol and bilirubin, revealed no statistically significant association, although there was a strong trend toward an association with change in $4 \beta$-hydroxycholesterol levels (Pearson $r=-0.572 ; P=$ 0.052) (Figure 6c,d). However, the increase in CYP2B6 expression in PBMCs was correlated with the reduction in AUC (Pearson $r=0.608, P=0.036$ ) (Figure 6f).

\section{DISCUSSION}

We report that long-term treatment with efavirenz in humans results in an induction of CAR target genes in PBMCs and the liver but has no impact on intestinal drug metabolism and transporters. Importantly, the study was originally designed to determine potential interactions of efavirenz with ezetimibe. Therefore, the data related to multiple-dose treatment with efavirenz were obtained in the presence of steady-state ezetimibe. However, testing for the impact of multiple doses of ezetimibe on hepatic markers and the expression of the target genes in PBMCs did not reveal any significant changes.

The induction of ABCB1 and CYP2B6 in PBMCs observed in the present study is in accordance with findings showing significant expression of CAR and PXR in these cells. ${ }^{27}$ However, in a previous study, Manceau et al. reported that there was no induction of ABCB1 after treatment of such cells with either the PXR inducer rifampin or the CAR inducer phenobarbital. The authors of the study also reported that ABCB1 was neither detectable nor induced; to explain the lack of induction, they cited very weak expression of the sensors in those cells. ${ }^{28}$ It is noteworthy that, although the outcomes with respect to gene expression are comparable for phenobarbital and efavirenz (thereby suggesting that both are CAR activators), it is well known that phenobarbital functions as an indirect activator leading to an increased nuclear translocation by activating intracellular signal cascades whereas efavirenz is assumed to activate the nuclear receptor directly by ligand binding. ${ }^{29}$ 
The phenobarbital-activated signal cascade has been identified in the liver and intestine, but it is not known whether the genes involved are present in PBMCs. In agreement with our findings, there are in vitro data showing an induction of $\mathrm{ABCB} 1$ in PBMCs after treatment with efavirenz. ${ }^{30}$ Efavirenz accumulates significantly in PBMCs both in vitro and in vivo, ${ }^{31}$ thereby supporting the idea that efavirenz may directly activate an intracellular sensor such as CAR. Our findings are in contrast to those recently reported by Burhenne et al., who found no significant induction of $\mathrm{ABCB} 1$ and $\mathrm{ABCC} 2$ expression in PBMCs. ${ }^{32}$ In our study, we were not able to detect mRNA expression of ABCC2 in PBMCs. The function of efflux transporters as part of a mechanism that modulates cellular accumulation of a compound, and thereby intracellular activity, continues to be a focus of pharmacologic research. In this context, Burhenne et al. report that cellular accumulation of efavirenz is independent of $\mathrm{ABCB} 1$ as determined by investigating the potential association between intracellular accumulation of efavirenz and the amount of ABCB1 expression. Similar results were obtained in cells isolated from patients with HIV ${ }^{26}$ At this point in time, there is no obvious explanation for the discrepancy between the in vivo observation by Burhenne et al. and our finding of induction of CAR target genes in PBMCs. However, one explanation for the conflicting data could be the presence of genetic variants. Even if functional, nonsynonymous variants of CAR occur very infrequently $(<5 \%)$, there is still the question of whether variants of regulatory genes are implicated in interindividual differences in response. Recent findings by Wyen et al. suggest an association between the synonymous variant rs2307424 (c.5719C >T, p.180Pro>Pro) and the clinical phenotype characterized by adverse neuropsychiatric side effects of efavirenz treatment, often leading to discontinuation of the drug. ${ }^{33}$

The issue of whether target gene expression in PBMCs or PBLs might be a surrogate marker for quantifying systemic (hepatic) enzyme activity has been widely discussed. There is convincing evidence that the amount of expression in PBMCs is not predictive of hepatic gene expression and activity per se. ${ }^{34}$ However, there is still a proposal to monitor modulation of mRNA expression to assess for in vivo PXR- or CAR-mediated changes in drug metabolism and transport. Our data provide evidence that the change in CYP2B6 expression is predictive of the change in efavirenz pharmacokinetics. Therefore, measuring the expression of genes in PBMCs might be a way to identify in vivo inducers without accessing hepatic liver tissue.

The discrepancy between the finding of hepatic induction of CAR-targeted genes and the absence of evidence of such induction in the intestine is in accordance with previous findings in rats, in which long-term treatment with efavirenz was reported to induce hepatic CYP3A-mediated midazolam metabolism without affecting ABCB1-mediated intestinal efflux of the probe substrate rhodamine 123 in everted gut sacs. ${ }^{35}$ Similar results were obtained by Mouly and colleagues in humans. They showed that long-term treatment with efavirenz results in induction of CYP3A4-mediated metabolism, as shown by monitoring using the sensitive erythromycin breath test, whereas no impact was observed on intestinal ABCB1 (P-gp) and CYP3A4 expression levels. ${ }^{36}$ In light of results from previous studies in the $\mathrm{CAR}^{-1-}$ mouse model and the well-known mouse-specific CAR inducer 1, 4-bis-[2-(3, 5,-dichloropyridyloxy)] benzene (TCPOBOP), it is well established that oral administration of a direct CAR ligand induces expression of these target genes in enterocytes. ${ }^{37}$ Similar results were obtained in testing the impact of TCPOBOP on expression of efflux transporters on the blood-brain barrier, ${ }^{38}$ suggesting that the observed phenomenon is specific for efavirenz.

It is noteworthy that responsiveness of intestinal CAR to activation by efavirenz is supported by in vitro findings in LS180 cells, a human intestinal cell line derived from a colon adenocarcinoma, showing increased expression and function of ABCB1 after efavirenz 
treatment. ${ }^{39,40}$ One explanation for our observation could be differential expressions of CAR splice variants in the intestine and liver. Indeed, several splice variants of CAR have been identified in humans, with one splice variant containing an in-frame insertion of five amino acids (APYLT) in the highly conserved region of the ligand-binding domain (LBD). According to reports by several independent groups, this variant exhibits minimal basal activity but potent ligand-induced activity. ${ }^{41,42}$ Importantly, Chen et al. reported in vitro findings that efavirenz exhibits high trans-activating activity toward the full-length CAR (CAR1) but negligible activity toward the variant containing the APYLT insertion. ${ }^{43}$ It is possible to speculate that differences in the expression profiles of these variants might explain our observation that there were no changes in intestinal gene expression whereas there were observable changes in plasma levels of $4 \beta$-hydroxycholesterol, a surrogate marker of CYP3A4 activity, ${ }^{24}$ as well as changes in efavirenz metabolism, which is mediated predominantly by CYP2B6. ${ }^{22,44}$ However, a comprehensive analysis of CAR splice variants revealed that the intestine exhibits less variability with regard to splicing than the liver does and, importantly, CAR1 is highly expressed in enterocytes. ${ }^{45,46}$ Similar results were obtained from the mRNA samples in our study (data not shown), suggesting that differences in the expression profiles of splice variants are unlikely to be the prominent reason for the phenotype reported here.

This leaves us with the speculation that the differences might be based on the pharmacokinetics of the lipophilic efavirenz, which undergoes very rapid absorption, with $T_{\max }$ being reached after only $2-3 \mathrm{~h}$ (Table 1 ). It is therefore possible to hypothesize that the intestinal transit of the drug is so rapid that intracellular concentrations in the enterocytes are too low for efficient CAR induction, suggesting that tissue-specific drug disposition is a determinant of the inductive capacity. We observed a similar phenomenon associated with tissue-specific induction in a previous study of the PXR inducer carbamazepine, in which induction was detected in the kidney and liver, with no effect on intestinal expression. ${ }^{47}$

Further studies are warranted to identify the underlying mechanism of the differential inductive capacity of efavirenz in different pharmacologic compartments.

\section{METHODS}

\section{Clinical study}

Study protocol-The in vivo data reported here are from samples obtained during a clinical study performed to evaluate the pharmacokinetic interaction between efavirenz and ezetimibe. The study protocol has been described in detail elsewhere. ${ }^{25}$ Briefly, after giving written informed consent, 12 healthy male subjects of Caucasian descent, between 20 and 36 years of age, were enrolled. The protocol included four study periods. Periods A and B were for investigation of the pharmacokinetics of a single-dose administration of efavirenz (400 $\mathrm{mg}$, Sustiva capsules; Bristol-Myers Squibb, Munich, Germany) and of steady-state ezetimibe $10 \mathrm{mg}$ (Ezetrol tablets; MSD Sharp \& Dohme, Haar, Germany), respectively. Period $\mathrm{C}$ was for assessing the pharmacokinetics of single-dose efavirenz plus steady-state ezetimibe, and period $\mathrm{D}$ was for determining the impact of chronic treatment with $400 \mathrm{mg}$ efavirenz once daily for 9 days on steady-state pharmacokinetics of ezetimibe. In each study period, the subjects received $400 \mathrm{mg}$ efavirenz, either alone or in combination with ezetimibe $(10 \mathrm{mg})$ as indicated, after an overnight fast for at least $10 \mathrm{~h}$. Venous blood samples $(7.5 \mathrm{ml})$ were collected from the forearm before administration of the treatment and at $0.5,1,1.5,2,2.5,3,3.5,4,5,6,8,10,12,16,24,36,48,72,96$, and $120 \mathrm{~h}$ after the administration, for quantitative analyses. Urine was collected in fractions of $24 \mathrm{~h}$ as indicated in Supplementary Figure S1 online. The study was approved by the local ethics committee of the Medical Faculty of the University of Greifswald and by the German Federal Institute for Drugs and Medical Devices (BfArM, 4035064) and was registered in 
the European (EudraCT 2009-011050-17) and US (ClinicalTrials.gov NCT00810303) clinical trial databases.

Quantitative assays for efavirenz in human plasma-Plasma efavirenz concentrations were measured using liquid chromatography-tandem mass spectrometry as previously reported. ${ }^{27}$ Plasma samples $(0.2 \mathrm{ml})$ were mixed with internal standard $(30 \mu \mathrm{l}$ of $500 \mathrm{ng} / \mathrm{ml}$ nevirapine) and extracted with $6 \mathrm{ml}$ ethyl acetate under alkaline $\mathrm{pH}(500 \mu \mathrm{l}$ of 1 $\mathrm{mol} / \mathrm{l}$ glycine $/ 1 \mathrm{~mol} / \mathrm{l}$ sodium hydroxide buffer, $\mathrm{pH} 11.3$ ). The extraction efficiency was $>80 \%$ across the standard curve. The samples were centrifuged at 3,600 rpm, and the organic layer was evaporated to dryness. The residues were reconstituted in $50 \mu 1$ of mobile phase. Volumes of $25 \mu \mathrm{l}$ were analyzed using the API 2000 tandem mass spectrometer (Applied Biosystems, Foster City, CA) equipped with a turbo ion spray and coupled with a Shimadzu HPLC system (Columbia, MD). Efavirenz and nevirapine were quantified using multiple-reaction monitoring at $\mathrm{m} / \mathrm{z}$ values of $316 / 244$ and 267/226, respectively, in positive mode. This assay had a lower limit of quantification of $1.0 \mathrm{ng} / \mathrm{ml}$, was linear over the range of 1.0 to $2,500 \mathrm{ng} / \mathrm{ml}$ efavirenz, and had within-day and between-day coefficients of $<15 \%$. Peaks consistent with ezetimibe and its glucuronide metabolite were not observed. Pharmacokinetic data were not modeled. All the pharmacokinetic values were directly generated from rough data. The maximum plasma concentrations $\left(C_{\max }\right)$ and the time point of $C_{\max }\left(T_{\max }\right)$ were taken from the concentration-time curves, and AUC was assessed using the trapezoidal rule. Renal clearance (CLR) was derived from the respective cumulative amounts $(\mathrm{Ae})$ of drugs excreted into urine over the range of the respective AUC. The elimination half-life values for the drugs were calculated by linear regression analysis of log serum concentration against time.

\section{Quantitative determination of bilirubin and $4 \beta$-hydroxycholesterol in human}

samples-The total bilirubin levels were measured photometrically, using the Dimension Vista System and a modification of the protocol originally published by Doumas et al. ${ }^{48}$ The analytical sensitivity of the assay for total bilirubin was $2 \mu \mathrm{mol} / \mathrm{l}(0.1 \mathrm{mg} / \mathrm{dl})$. The concentration of $4 \beta$-hydroxycholesterol was determined using a highly sensitive and specific isotope-dilution method involving gas chromatography-mass spectrometry, recently described by Lütjohann et al. ${ }^{49}$ The limit of quantification was $3.0 \mathrm{ng} / \mathrm{ml}$, and the linear range was 3.0-400 ng/ml. Between- and within-day precision values were 2.1 and $2.7 \%$, respectively, and the between- and within-day accuracy percentages were 2.0 and $3.3 \%$, respectively.

Duodenal biopsy samples-Duodenal samples were obtained from healthy subjects via gastroduodenoscopy in the Clinic of Internal Medicine of the University Hospital Greifswald. Biopsy samples were obtained 3 days before the first study day and on study day 29 , i.e., 9 days after chronic administration of efavirenz. The duodenal samples were immediately snap-frozen in liquid nitrogen, either diluted in $1.0 \mathrm{ml}$ RNAlater solution (Qiagen, Hilden, Germany) for RNA isolation or without any supplementation for protein isolation. Subsequently, the samples were stored at $-80^{\circ} \mathrm{C}$ until further handling. Total mRNA was isolated using the RNeasy Mini Kit in accordance with the manufacturer's (Qiagen) instructions. Determinations of integrity and amount of mRNA were performed using the Lab-on-a-Chip technology and the Agilent Bioanalyzer (Agilent, Santa Clara, CA).

Isolation of PBMCs-PBMCs were isolated from EDTA blood samples ( $5 \mathrm{ml})$ drawn during the first $3 \mathrm{~h}$ of the pharmacokinetic analysis of periods $\mathrm{A}, \mathrm{B}$, and $\mathrm{D}$. The blood samples were immediately centrifuged at $1,500 \mathrm{~g}$ at $4{ }^{\circ} \mathrm{C}$ for $15 \mathrm{~min}$. The resulting buffy coat was transferred to a falcon tube and stored on ice. After all samples from an individual were 
pooled, the buffy coat was diluted in ice-cold phosphate-buffered saline (total volume of 30 $\mathrm{ml})$. For the enrichment of PBMCs, the diluted buffy coat was placed on $15 \mathrm{ml}$ Lymphoprep (Axis-Shield, Oslo, Norway) and centrifuged for $30 \mathrm{~min}$ at $700 \mathrm{~g}$ at room temperature, using a swing-out rotor. The resulting cell band was collected to a new falcon tube and washed several times with $0.9 \% \mathrm{NaCl}$. After the cells were counted using a CASY1 cell counter (Schaerfe System, Reutlingen, Germany), aliquots of $2.5 \times 10^{6}$ cells were stored in liquid nitrogen. Two aliquots were used for subsequent RNA isolation using the RNeasy Mini Kit (Qiagen) as described earlier.

PBMC culture-Isolated PBMCs from treatment-naive healthy volunteers were cultured in RPBMI-1640 supplemented with $10 \%$ heat-in-activated FCS, $1 \%$ sodium pyruvate, and $1 \% \mathrm{~L}$-glutamine. After a 24 -h adaptation period, efavirenz $(10 \mu \mathrm{mol} / \mathrm{l})$ or dimethyl sulfoxide control was added to the culture medium. After $48 \mathrm{~h}$ of incubation, the cells were harvested and total mRNA was isolated using the RNeasy Mini Kit (Qiagen).

In vitro treatment of primary human hepatocytes-Primary human hepatocytes from three individuals were purchased from Lonza Verviers SPRL (Verviers, Belgium). HMM medium supplemented with the Lonza HCM bullet was added to the plated cells, and they were incubated for $24 \mathrm{~h}$ at $37{ }^{\circ} \mathrm{C}$ in a humidified atmosphere containing $5 \% \mathrm{CO}_{2}$. The cells were treated with efavirenz $(1 \mu \mathrm{mol} / \mathrm{l})$, CITCO $(1 \mu \mathrm{mol} / \mathrm{l})$, or dimethyl sulfoxide control. After $48 \mathrm{~h}$ of incubation, the cells were harvested and RNA was isolated.

In vitro treatment of CAR-transfected Caco2 cells-Caco2 cells were transiently transfected with hCAR-pEF6-V5/HIS. In brief, Caco2 cells were placed in 12-well plates; once $80 \%$ confluence was reached, the cells were transfected with $350 \mathrm{ng}$ of the plasmid, using $3 \mu 1$ Lipofectin (Invitrogen Life Technologies, Darmstadt, Germany).The following day, the cells were treated with efavirenz ( 1 or $10 \mu \mathrm{mol} / \mathrm{l})$, CITCO $(1 \mu \mathrm{mol} / \mathrm{l})$ (SigmaAldrich, St. Louis, MO) or dimethyl sulfoxide control diluted in OptiMEM as culture medium (Invitrogen). After $24 \mathrm{~h}$ of incubation, the cells were harvested, and total mRNA was isolated.

Determination of $\mathbf{m R n A}$ expression-For purposes of real-time PCR, 1,500 ng of total RNA was reverse-transcribed using the TaqMan reverse transcription kit (Applied Biosystems, Darmstadt, Germany) followed by quantitative real-time PCR. Gene expression levels of villin, 18S, CAR, ABCB1, ABCC2, CYP3A4, CYP2B6, UGT1A1, UGT1A3, UGT2B7, and UGT2B15 were determined using predeveloped TaqMan assays, TaqMan Gene Expression Master Mix, and the 7900HT fast real-time PCR system (Applied Biosystems). The mRNA expressions of genes from intestinal biopsy samples obtained before and after chronic treatment with efavirenz ( $400 \mathrm{mg} / \mathrm{p}$. . . day) and those obtained after in vitro treatment of the cells were normalized to that of 18S-rRNA in the same sample and to the mean of normalized gene expressions in all pretreatment samples or control treatments, respectively, using the $\Delta / \Delta$-Ct method as previously described. ${ }^{50}$ For semiquantitative determination of mRNA expressions, PCR was performed using the predeveloped TaqMan assays and a Veriti thermal cycler (Applied Biosystems) as described above. PCR was stopped after 37 cycles, and gene expression was visualized after electrophoresis using the Gel Logic 200 imaging system (Raytest, Straubenhardt, Germany).

Western blot analysis-Proteins were isolated from the duodenal tissue samples. Briefly, the biopsy samples were transferred to a tube containing $250 \mu 15 \mathrm{mmol} / \mathrm{l}$ Tris- $\mathrm{HCl}$ (pH 7.5) supplemented with protease inhibitors $(0.3 \mu \mathrm{mol} / 1$ aprotinin, $1 \mu \mathrm{mol} / \mathrm{l}$ leupeptin, and $0.1 \mathrm{mmol} / \mathrm{l}$ phenylmethylsulfonyl fluoride). After mechanical dissection using a small pair of scissors, the tissue was homogenized on ice using a Dounce Potter. After 15 min of 
centrifugation at $9,000 \mathrm{~g}$ at $4{ }^{\circ} \mathrm{C}$, the protein content in the supernatant was determined using a bichinonic acid assay (Sigma-Aldrich). A quantity of $50 \mu \mathrm{g}$ of protein was used for western blot analysis. SDS-PAGE proteins were electrotransferred to nitrocellulose using a tank blotting system (Bio-RAD, Munich, Germany). Before incubation with the primary antibody, the membrane was blocked with 5\% skim milk diluted in Tris-buffered saline plus Tween-20. The specific primary antibody was diluted in Tris-buffered saline plus Tween-20 and $0.1 \%$ bovine serum albumin and incubated overnight at $4{ }^{\circ} \mathrm{C}$. For detection of UGT1A1 and CYP3A4, polyclonal rabbit sera anti-UGT1A1 (Sigma-Aldrich) and anti-CYP3A4 (ab22704, abcam, Cambridge, UK), respectively, were used. The efflux transporters $\mathrm{ABCB} 1, \mathrm{ABCG} 2$, and $\mathrm{ABCC} 2$ were detected using the mouse mono-clonal antibodies $\mathrm{C} 219$ (Calbiochem, Darmstadt, Germany), BXP-21 (Alexis Biochemicals, Loerrach, Germany), and M2 III-6 (Alexis Biochemicals). UGT2B isoforms were detected using a goat polyclonal antibody (sc-23479, Santa Cruz, CA). Binding of the primary antibody was detected using the respective horseradish peroxidase-labeled secondary antibody (Vector Laboratories, Burlington, ON, Canada) and ECL Plus Western Blot Detection reagent (GE Healthcare, Freiburg, Germany). Chemiluminescence was detected after incubating the blot for $1 \mathrm{~min}$ with $1 \mathrm{ml}$ of the detection reagent. Images were recorded at variable exposure times, using the Molecular Imager Chemi Doc TM XRS imaging system and the Quantity One software 1D (Bio-RAD, Munich, Germany).

Statistical analysis-Gene expression data from in vivo and in vitro studies were analyzed for statistical significance by means of the Mann-Whitney test and Student's $t$-test, respectively. Significance was assumed if $P$ values were $<0.05$. The Wilcoxon rank sum test was performed to compare the pharmacokinetic parameters of efavirenz with the levels of $4 \beta$-hydroxycholesterol and total bilirubin in plasma. To avoid $a$-error accumulation by multiple testing, the Bonferroni method was used. Statistical significance was assumed for $P$ values $<0.017$. Statistical analyses were performed using GraphPad Prism (version 5.04, La Jolla, CA), and $P$ values $<0.05$ were considered statistically significant.

\section{Supplementary Material}

Refer to Web version on PubMed Central for supplementary material.

\section{Acknowledgments}

This work was supported by the GANI MED project grant (Greifswald Approach to Individualized Medicine, grant 03IS2061A) funded by the German Federal Ministry for Education and Research (Bundesministerium für Bildung und Forschung, BMBF), by an InnoProfile Project grant (grant 03IP612, InnoProfile), and by the National Institute of General Medical Sciences, National Institutes of Health, Bethesda, MD (grants RO1GM078501 and 3R01GM078501-04S1) (Z.D.)

\section{References}

1. Lehmann JM, McKee DD, Watson MA, Willson TM, Moore JT, Kliewer SA. The human orphan nuclear receptor PXR is activated by compounds that regulate CYP3A4 gene expression and cause drug interactions. J. Clin. Invest. 1998; 102:1016-1023. [PubMed: 9727070]

2. Goodwin B, Moore LB, Stoltz CM, McKee DD, Kliewer SA. Regulation of the human CYP2B6 gene by the nuclear pregnane X receptor. Mol. Pharmacol. 2001; 60:427-431. [PubMed: 11502872]

3. Gerbal-Chaloin S, Daujat M, Pascussi JM, Pichard-Garcia L, Vilarem MJ, Maurel P. Transcriptional regulation of CYP2C9 gene. Role of glucocorticoid receptor and constitutive androstane receptor. J. Biol. Chem. 2002; 277:209-217. [PubMed: 11679585]

4. Gardner-Stephen D, et al. Human PXR variants and their differential effects on the regulation of human UDP-glucuronosyltransferase gene expression. Drug Metab. Dispos. 2004; 32:340-347. [PubMed: 14977869] 
5. Sugatani J, et al. The induction of human UDP-glucuronosyltransferase 1A1 mediated through a distal enhancer module by flavonoids and xenobiotics. Biochem. Pharmacol. 2004; 67:989-1000. [PubMed: 15104253]

6. Sonoda J, Xie W, Rosenfeld JM, Barwick JL, Guzelian PS, Evans RM. Regulation of a xenobiotic sulfonation cascade by nuclear pregnane X receptor (PXR). Proc. Natl. Acad. Sci. USA. 2002; 99:13801-13806. [PubMed: 12370413]

7. Alnouti Y, Klaassen CD. Regulation of sulfotransferase enzymes by prototypical microsomal enzyme inducers in mice. J. Pharmacol. Exp. Ther. 2008; 324:612-621. [PubMed: 17993606]

8. Meyer Zu Schwabedissen HE, Tirona RG, Yip CS, Ho RH, Kim RB. Interplay between the nuclear receptor pregnane $\mathrm{X}$ receptor and the uptake transporter organic anion transporter polypeptide 1A2 selectively enhances estrogen effects in breast cancer. Cancer. Res. 2008; 68:9338-9347. [PubMed: 19010908]

9. Geick A, Eichelbaum M, Burk O. Nuclear receptor response elements mediate induction of intestinal MDR1 by rifampin. J. Biol. Chem. 2001; 276:14581-14587. [PubMed: 11297522]

10. Wang H, LeCluyse EL. Role of orphan nuclear receptors in the regulation of drug-metabolising enzymes. Clin. Pharmacokinet. 2003; 42:1331-1357. [PubMed: 14674787]

11. Moore LB, et al. St. John's wort induces hepatic drug metabolism through activation of the pregnane X receptor. Proc. Natl. Acad. Sci. USA. 2000; 97:7500-7502. [PubMed: 10852961]

12. Borrelli F, Izzo AA. Herb-drug interactions with St John's wort (Hypericum perforatum): an update on clinical observations. AAPS J. 2009; 11:710-727. [PubMed: 19859815]

13. Burk O, Arnold KA, Geick A, Tegude H, Eichelbaum M. A role for constitutive androstane receptor in the regulation of human intestinal MDR1 expression. Biol. Chem. 2005; 386:503-513. [PubMed: 16006237]

14. Gerbal-Chaloin S, Daujat M, Pascussi JM, Pichard-Garcia L, Vilarem MJ, Maurel P. Transcriptional regulation of CYP2C9 gene. Role of glucocorticoid receptor and constitutive androstane receptor. J. Biol. Chem. 2002; 277:209-217. [PubMed: 11679585]

15. Sueyoshi T, Kawamoto T, Zelko I, Honkakoski P, Negishi M. The repressed nuclear receptor CAR responds to phenobarbital in activating the human CYP2B6 gene. J. Biol. Chem. 1999; 274:60436046. [PubMed: 10037683]

16. Sugatani J, et al. The phenobarbital response enhancer module in the human bilirubin UDPglucuronosyltransferase UGT1A1 gene and regulation by the nuclear receptor CAR. Hepatology. 2001; 33:1232-1238. [PubMed: 11343253]

17. Smirlis D, Muangmoonchai R, Edwards M, Phillips IR, Shephard EA. Orphan receptor promiscuity in the induction of cytochromes p450 by xenobiotics. J. Biol. Chem. 2001; 276:12822-12826. [PubMed: 11278292]

18. Baes M, Gulick T, Choi HS, Martinoli MG, Simha D, Moore DD. A new orphan member of the nuclear hormone receptor superfamily that interacts with a subset of retinoic acid response elements. Mol. Cell. Biol. 1994; 14:1544-1552. [PubMed: 8114692]

19. Maglich JM, et al. Identification of a novel human constitutive androstane receptor (CAR) agonist and its use in the identification of CAR target genes. J. Biol. Chem. 2003; 278:17277-17283. [PubMed: 12611900]

20. Kobayashi K, et al. Identification of HMG-CoA reductase inhibitors as activators for human, mouse and rat constitutive androstane receptor. Drug Metab. Dispos. 2005; 33:924-929. [PubMed: 15802384]

21. Faucette SR, et al. Relative activation of human pregnane $X$ receptor versus constitutive androstane receptor defines distinct classes of CYP2B6 and CYP3A4 inducers. J. Pharmacol. Exp. Ther. 2007; 320:72-80. [PubMed: 17041008]

22. Ngaimisi E, et al. Long-term efavirenz autoinduction and its effect on plasma exposure in HIV patients. Clin. Pharmacol. Ther. 2010; 88:676-684. [PubMed: 20881953]

23. Gerber JG, et al. AIDS Clinical Trials Group A5108 Team. Effect of efavirenz on the pharmacokinetics of simvastatin, atorvastatin, and pravastatin: results of AIDS Clinical Trials Group 5108 Study. J. Acquir. Immune Defic. Syndr. 2005; 39:307-312. [PubMed: 15980690]

24. Diczfalusy U, Kanebratt KP, Bredberg E, Andersson TB, Böttiger Y, Bertilsson L. 4betahydroxycholesterol as an endogenous marker for CYP3A4/5 activity. Stability and half-life of 
elimination after induction with rifampicin. Br. J. Clin. Pharmacol. 2009; 67:38-43. [PubMed: 19006545]

25. Oswald $\mathrm{S}$, et al. Impact of efavirenz on intestinal metabolism and transport: insights from an interaction study with ezetimibe in healthy volunteers. Clin. Pharmacol.Ther. 2012; 91:506-513. [PubMed: 22297387]

26. Almond LM, Hoggard PG, Edirisinghe D, Khoo SH, Back DJ. Intracellular and plasma pharmacokinetics of efavirenz in HIV-infected individuals. J. Antimicrob. Chemother. 2005; 56:738-744. [PubMed: 16141277]

27. Siest G, et al. Transcription factor and drug-metabolizing enzyme gene expression in lymphocytes from healthy human subjects. Drug Metab. Dispos. 2008; 36:182-189. [PubMed: 17940135]

28. Manceau S, et al. Lack of P-glycoprotein induction by rifampicin and phenobarbital in human lymphocytes. Int. J. Pharm. 2010; 395:98-103. [PubMed: 20488228]

29. Goodwin B, Moore JT. CAR: detailing new models. Trends Pharmacol. Sci. 2004; 25:437-441. [PubMed: 15276713]

30. Chandler B, et al. The effects of protease inhibitors and nonnucleoside reverse transcriptase inhibitors on p-glycoprotein expression in peripheral blood mononuclear cells in vitro. J. Acquir. Immune Defic. Syndr. 2003; 33:551-556. [PubMed: 12902797]

31. Janneh $\mathrm{O}$, et al. Intracellular accumulation of efavirenz and nevirapine is independent of Pglycoprotein activity in cultured CD4 T cells and primary human lymphocytes. J. Antimicrob. Chemother. 2009; 64:1002-1007. [PubMed: 19748977]

32. Burhenne $\mathrm{J}$, et al. No evidence for induction of $\mathrm{ABC}$ transporters in peripheral blood mononuclear cells in humans after 14 days of efavirenz treatment. Antimicrob. Agents Chemother. 2010; 54:4185-4191. [PubMed: 20660679]

33. Wyen C, et al. German Competence Network for HIV/AIDS Coordinators. Cytochrome P450 $2 \mathrm{~B} 6$ (CYP2B6) and constitutive androstane receptor (CAR) polymorphisms are associated with early discontinuation of efavirenz-containing regimens. J. Antimicrob. Chemother. 2011; 66:20922098. [PubMed: 21715435]

34. Albermann N, et al. Expression of the drug transporters MDR1/ABCB1, MRP1/ABCC1, MRP2/ ABCC2, BCRP/ABCG2, and PXR in peripheral blood mononuclear cells and their relationship with the expression in intestine and liver. Biochem. Pharmacol. 2005; 70:949-958. [PubMed: 16054595]

35. Berruet N, Sentenac S, Auchere D, Gimenez F, Farinotti R, Fernandez C. Effect of efavirenz on intestinal p-glycoprotein and hepatic p450 function in rats. J. Pharm. Pharm. Sci. 2005; 8:226-234. [PubMed: 16124934]

36. Mouly S, et al. Hepatic but not intestinal CYP3A4 displays dose-dependent induction by efavirenz in humans. Clin. Pharmacol. Ther. 2002; 72:1-9. [PubMed: 12151999]

37. Wei P, Zhang J, Dowhan DH, Han Y, Moore DD. Specific and overlapping functions of the nuclear hormone receptors CAR and PXR in xenobiotic response. Pharmacogenomics J. 2002; 2:117-126. [PubMed: 12049174]

38. Wang X, Sykes DB, Miller DS. Constitutive androstane receptor-mediated up-regulation of ATPdriven xenobiotic efflux transporters at the blood-brain barrier. Mol. Pharmacol. 2010; 78:376383. [PubMed: 20547735]

39. Weiss J, Herzog M, König S, Storch CH, Ketabi-Kiyanvash N, Haefeli WE. Induction of multiple drug transporters by efavirenz. J. Pharmacol. Sci. 2009; 109:242-250. [PubMed: 19234366]

40. Störmer E, von Moltke LL, Perloff MD, Greenblatt DJ. Differential modulation of P-glycoprotein expression and activity by non-nucleoside HIV-1 reverse transcriptase inhibitors in cell culture. Pharm. Res. 2002; 19:1038-1045. [PubMed: 12180537]

41. Auerbach SS, Ramsden R, Stoner MA, Verlinde C, Hassett C, Omiecinski CJ. Alternatively spliced isoforms of the human constitutive androstane receptor. Nucleic Acids Res. 2003; 31:3194-3207. [PubMed: 12799447]

42. Jinno $\mathrm{H}$, et al. Identification of novel alternative splice variants of human constitutive androstane receptor and characterization of their expression in the liver. Mol. Pharmacol. 2004; 65:496-502. [PubMed: 14978227] 
43. Chen T, et al. A single amino acid controls the functional switch of human constitutive androstane receptor (CAR) 1 to the xenobiotic-sensitive splicing variant CAR3. J. Pharmacol. Exp. Ther. 2010; 332:106-115. [PubMed: 19820207]

44. Smith PF, DiCenzo R, Morse GD. Clinical pharmacokinetics of non-nucleoside reverse transcriptase inhibitors. Clin. Pharmacokinet. 2001; 40:893-905. [PubMed: 11735608]

45. Lamba JK, et al. Expression of constitutive androstane receptor splice variants in human tissues and their functional consequences. J. Pharmacol. Exp. Ther. 2004; 311:811-821. [PubMed: 15194709]

46. Arnold KA, Eichelbaum M, Burk O. Alternative splicing affects the function and tissue-specific expression of the human constitutive androstane receptor. Nucl. Recept. 2004; 2:1. [PubMed: 15043764]

47. Giessmann T, et al. Carbamazepine regulates intestinal P-glycoprotein and multidrug resistance protein MRP2 and influences disposition of talinolol in humans. Clin. Pharmacol. Ther. 2004; 76:192-200. [PubMed: 15371980]

48. Doumas BT, Wu TW. The measurement of bilirubin fractions in serum. Crit. Rev. Clin. Lab. Sci. 1991; 28:415-445. [PubMed: 1772588]

49. Lütjohann D, et al. 4beta-hydroxycholesterol as a marker of CYP3A4 inhibition in vivo - effects of itraconazole in man. Int. J. Clin. Pharmacol. Ther. 2009; 47:709-715. [PubMed: 19954708]

50. Schmittgen TD, Livak KJ. Analyzing real-time PCR data by the comparative C(T) method. Nat. Protoc. 2008; 3:1101-1108. [PubMed: 18546601] 


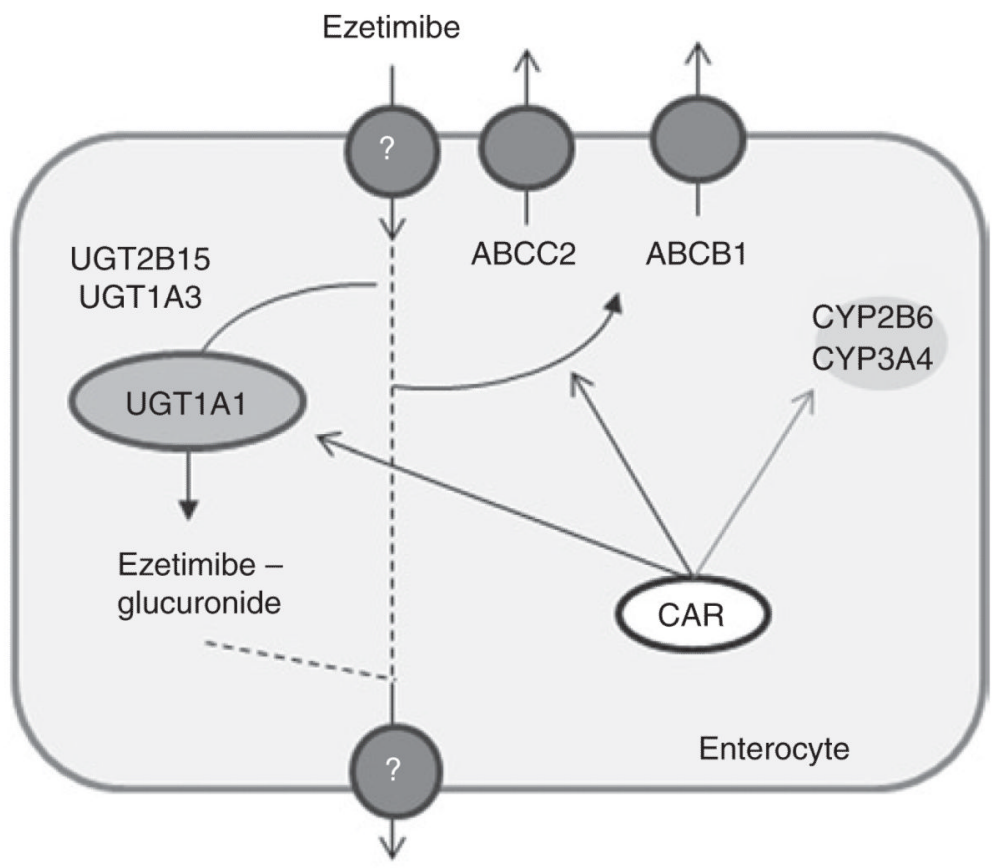

Figure 1.

Schematic of the potential CAR target genes involved in the intestinal handling of ezetimibe. CAR, constitutive androstane receptor. 
a



b

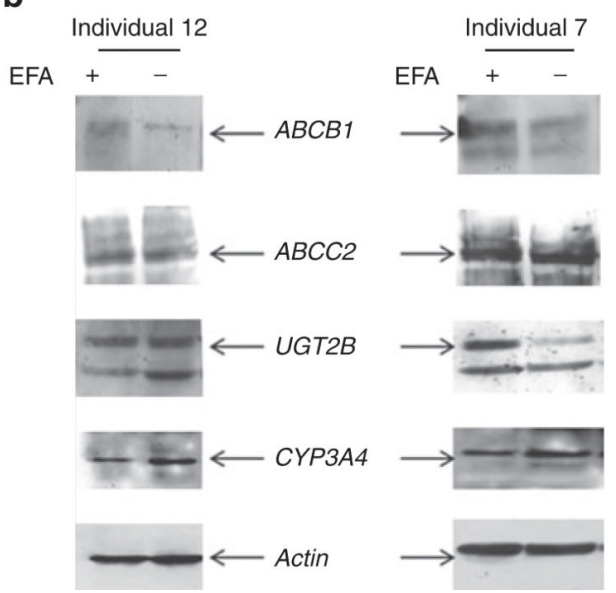

Figure 2.

Protein expression in human intestinal samples before and after long-term treatment with efavirenz (400 mg/day). (a) Expression levels of the CAR target genes UGT1A1 and $A B C G 2$ were determined by western blot analysis of duodenal biopsies obtained from healthy individuals $(n=8)$. (b) Expression levels of $A B C B 1, A B C C 2, U G T 2 B$, and $C Y P 3 A 4$ were determined in individuals 7 and 12 of the clinical study. The amount of actin expression was determined as a control for assessing protein loading. CAR, constitutive androstane receptor; EFA, efavirenz. 
a

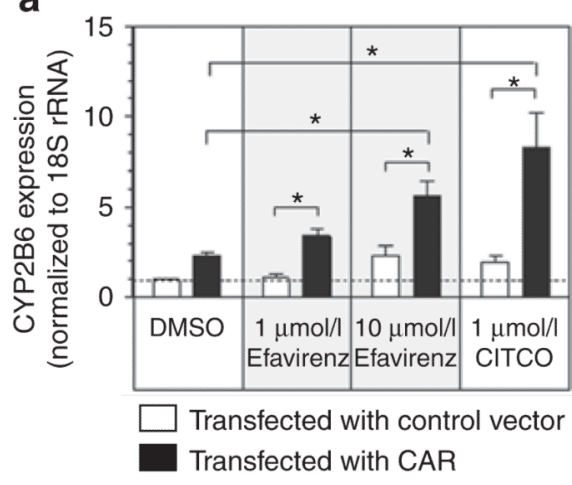

b

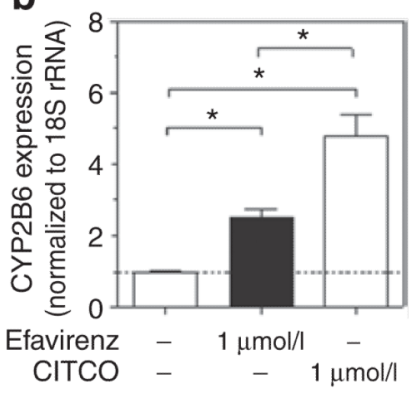

Figure 3.

CAR-mediated induction of CYP2B6 in (a) a human intestinal cell line (Caco2) and (b) freshly isolated human hepatocytes. Before treatment, Caco2 cells were transfected with the coding sequence of CAR or a control vector (pEF6-V5/His). After $24 \mathrm{~h}$ of incubation and subsequent treatment for $24 \mathrm{~h}$ with efavirenz $(1 \mu \mathrm{mol} / \mathrm{l}$ or $10 \mu \mathrm{mol} / \mathrm{l})$, CITCO $(1 \mu \mathrm{mol} / \mathrm{l})$, or DMSO control, the cells were harvested for mRNA quantification by means of real-time PCR. CYP2B6 mRNA expression was determined $24 \mathrm{~h}$ after the treatment of freshly isolated human hepatocytes. The data are presented as mean multiple increases in induction \pm SEM, relative to the DMSO control. $* P<0.05$ Student's $t$-test. CAR, constitutive androstane receptor; CITCO, imidazole-derivate 6-(4-chlorophenyl)imidazo[2,1- $\beta]$ [1,3]thiazole-5-carbaldehyde-O-(3,4-dichlorobenzyl)oxime; DMSO, dimethyl sulfoxide. 


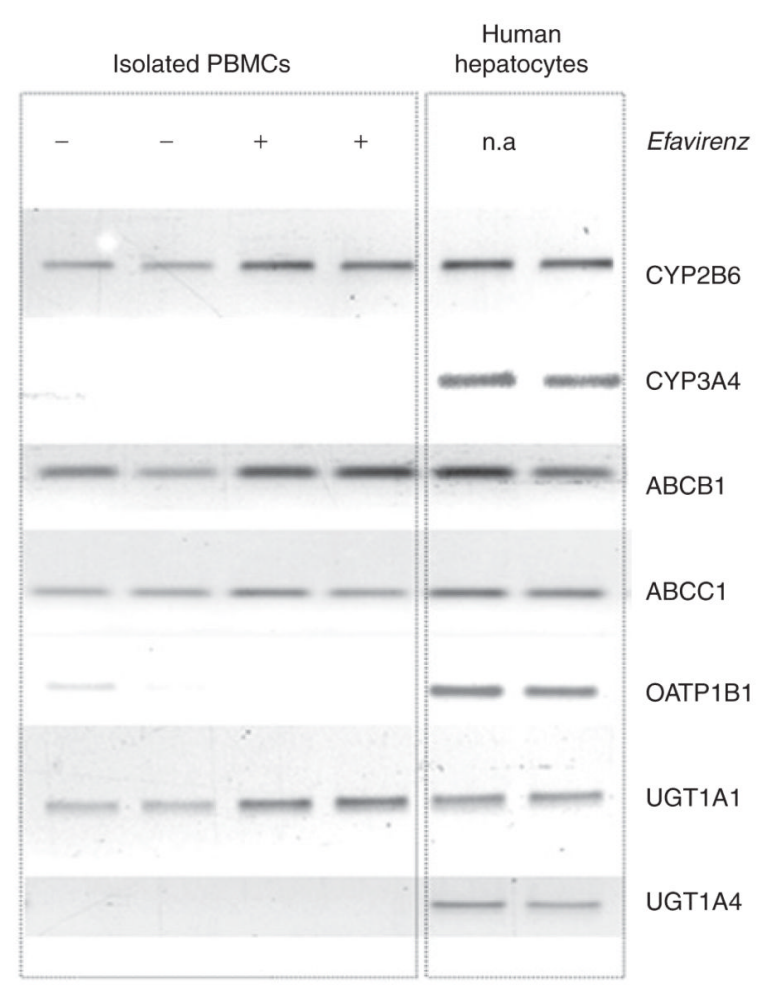

Figure 4.

Influence of efavirenz on CAR target gene expression in human PBMCs in vitro. PBMCs were isolated and treated with $10 \mu \mathrm{mol} / 1$ efavirenz in vitro. After $48 \mathrm{~h}$ of incubation, the cells were harvested, and the expression levels of known target genes were determined by means of semiquantitative PCR. The analysis showed significant induction of $C Y P 2 B 6$, $A B C B 1$, and $U G T 1 A 1$. Expression profiles of efavirenz in primary human hepatocytes served as positive controls. CAR, constitutive androstane receptor; n.a., not applicable; PBMC, peripheral blood mononuclear cell. 



Figure 5.

Gene expression in human PBMCs isolated from individuals on days designated for pharmacokinetic study. PBMCs were isolated during the first $3 \mathrm{~h}$ of the days on which pharmacokinetic studies were scheduled to measure parameters after a single dose of efavirenz (treatment period A), multiple doses of ezetimibe (treatment period B), and multiple doses of ezetimibe plus multiple doses of efavirenz (treatment period D). The expression levels of $C Y P 2 B 6, U G T 1 A 1, A B C B 1$, and $A B C C 1$ were determined by means of real-time PCR, and normalized to the value obtained for 18S-rRNA. $* P<0.05$, Students $t$-test. PBMC, peripheral blood mononuclear cell. 
a



C

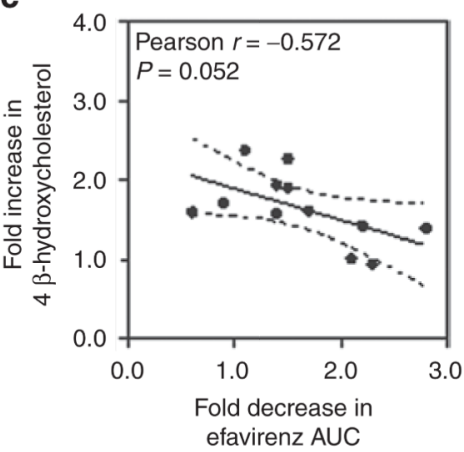

b

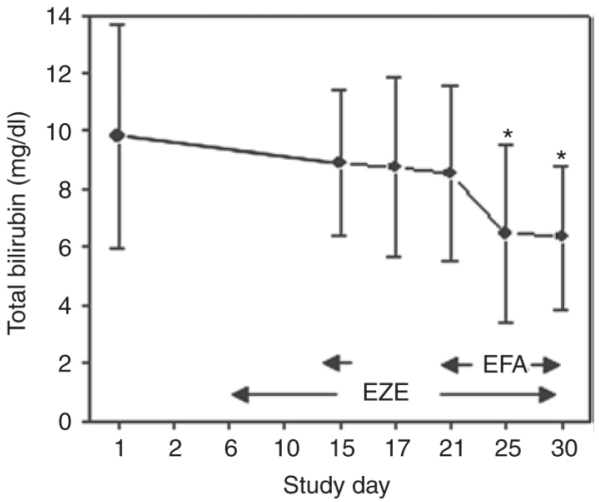

d

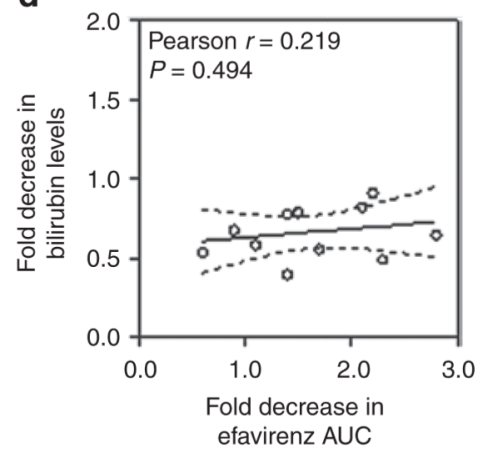

e



Figure 6.

Determination of endogenous markers of hepatic metabolism. $(\mathbf{a}, \mathbf{b})$ The ratio of $4 \beta$ hydroxycholesterol/cholesterol and the plasma bilirubin levels were determined at several time points. ${ }^{*} P<0.05$, ANOVA, $n=12$. The changes in (c) $4 \beta$-hydroxycholesterol and (d) total bilirubin levels and the change in (e) CYP2B6 expression in PBMCs were correlated with changes in efavirenz AUC assessed after a single dose and during steady state, respectively. Pearson regression; $P$ value of F test. AUC, area under the curve; EFA, efavirenz; EZE, ezetimibe; PBMC, peripheral blood mononuclear cell. 


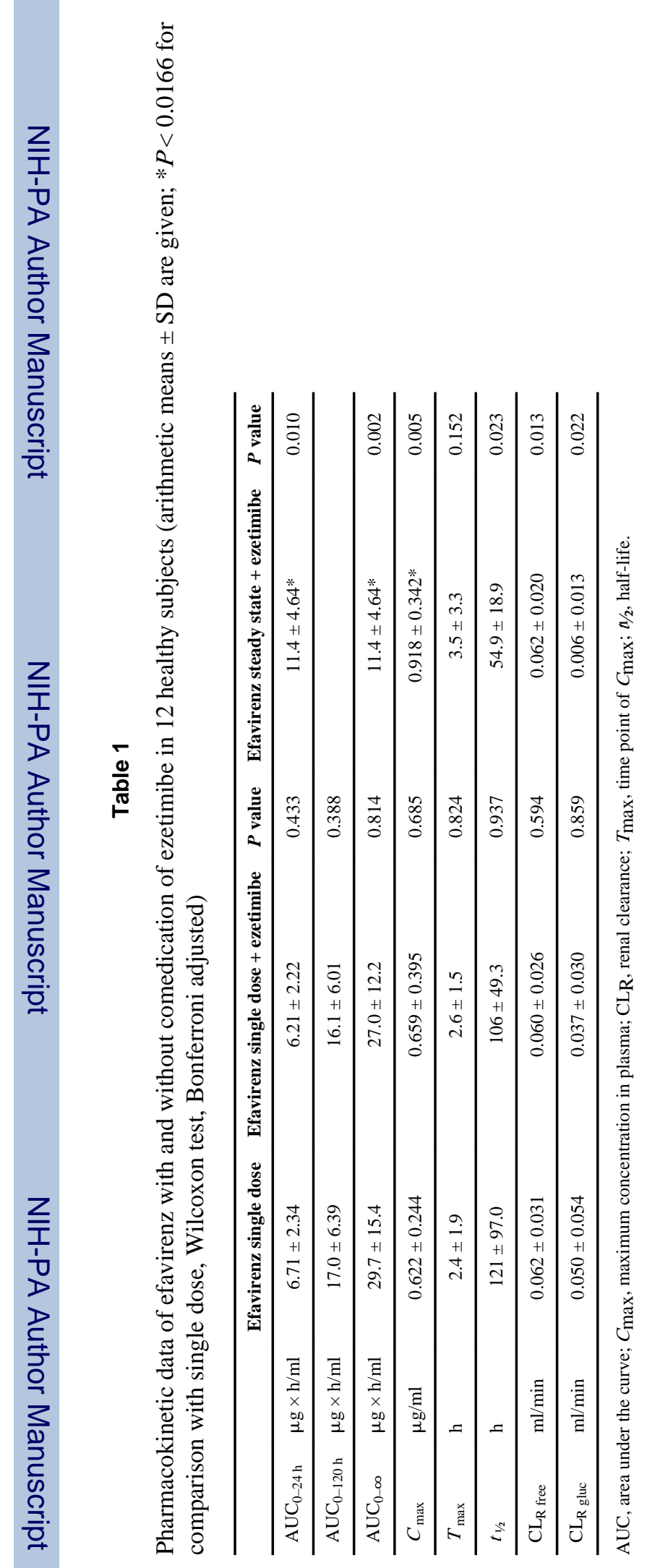

